\title{
AVALIAÇÃO DA EXATIDÃO E DA PRECISÃO DE MÉTODOS DE ANÁLISE DE RESÍDUOS DE PESTICIDAS MEDIANTE ENSAIOS DE RECUPERAÇÃO
}

\author{
NATILENE MESQUITA BRITO * \\ OZELITO POSSIDÔNIO DE AMARANTE JUNIOR ** \\ LUCIANA POLESE \\ TERESA CRISTINA RODRIGUES DOS SANTOS **** \\ MARIA LÚCIA RIBEIRO *****
}

\begin{abstract}
Discute-se a importância da exatidão e da precisão na validação de métodos de análise de resíduos de pesticidas. São apresentados, mediante dois estudos de casos, os principais processos para a avaliação da exatidão. Nos casos relatados foram obtidas recuperações entre 80 e $104 \%$ e coeficiente de variação entre 2,81 e $12,74 \%$, ambos enquadrados na faixa recomendada pela Association of Official Analytical Chemists (AOAC) para os níveis de concentração investigados. Os estudos evidenciaram que, os ensaios de recuperação são adequados para a avaliação dos critérios de exatidão e precisão para as mesmas matrizes.
\end{abstract}

PALAVRAS-CHAVE: MÉTODOS DE ANÁLISE-VALIDAÇÃO; PESTICIDAS-ANÁLISE.

\section{INTRODUÇÃO}

O desenvolvimento de métodos constitui etapa extremamente relevante na área de análise de resíduos de pesticidas. A investigação deve sempre incluir a validação do método desenvolvido e não apenas sua otimização, principalmente quando envolve sua aplicação em determinado tipo de matriz e não apenas estudos com soluções padrão (1). A validação de métodos analíticos tem sido discutida em diversos

* Mestrando em Química, Universidade Estadual Paulista "Júlio de Mesquita Filho" (UNESP), Araraquara - SP. (e-mail: natibrit@posgrad.iq.unesp.br).

** Mestrando em Química Analítica, Universidade Federal do Maranhão (UFMA), São Luís, MA.

*** Doutora em Química, Instituto de Química, UNESP, Araraquara - SP.

**** Professora, Doutora em Química Analítica, Departamento de Tecnologia Química, CCET, UFMA, São Luís - MA.

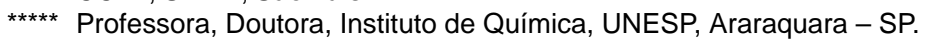


trabalhos na literatura (2-11). O enfoque dado na avaliação de métodos analíticos varia em função da área de aplicação, da concentração do analito, do objetivo do estudo e da natureza do método (12-17).

O processo de validação consiste na avaliação de diversos critérios, ou seja, seletividade, linearidade, gráfico analítico, sensibilidade, limites de detecção e quantificação, exatidão, precisão e consistência dos dados gerados (18). Dentre os critérios citados, a exatidão e a precisão são considerados os mais relevantes porque permitem estimar os erros e variações embutidos nos resultados analíticos. A exatidão pode ser estimada pelo cálculo do erro absoluto e relativo e a precisão pelas medidas de dispersão, como desvio-padrão, variância e coeficiente de variação, entre outras (3). A precisão costuma ser expressa como repetitividade, precisão intermediária ou reprodutividade (1). A repetitividade constitui a precisão estudada no mesmo laboratório, em pequeno intervalo de tempo (mesmo dia, analista e equipamento). A precisão intermediária é expressa pela variação entre resultados obtidos em dias diferentes pelo mesmo laboratório. A reprodutividade é estudada entre diferentes laboratórios, em diversas localidades do mundo (18), utilizando o mesmo conjunto de amostras.

Em análise de resíduos de pesticidas a validação do método ocorre, usualmente, por meio de ensaios de recuperação (19), uma vez que esse processo gera todas as informações necessárias para a avaliação do procedimento analítico (18). Além disto, são realizados no próprio laboratório, sem necessidade de obtenção de materiais de referência certificados (MRC), adesão de outros laboratórios ou existência prévia de métodos validados para o analito e a matriz em estudo (18).

Para determinar os parâmetros indispensáveis à validação são necessárias amostras contendo o analito de interesse em concentrações conhecidas. Essas amostras, no estudo de resíduos de pesticidas, são obtidas adicionando-se os compostos a serem analisados na amostra controle, também chamada amostra testemunha.

Denomina-se "nível de fortificação" a concentração em que o analito é adicionado na amostra controle. A escolha do nível de fortificação depende do propósito do estudo a ser realizado. Em análise de resíduos de pesticidas em alimentos toma-se como base os Limites Máximos de Resíduos (LMR), estabelecidos pela legislação nacional e internacional para cada analito e matriz. O procedimento de fortificação realizado in vitro pode não reproduzir a amostra em que o analito está quimicamente ligado aos constituintes da matriz. Assim sendo, na adição do analito à amostra deve-se considerar alguns fatores, tais como: tipo de matriz, tempo de fortificação e volume de solução padrão do pesticida.

A recuperação consiste na relação percentual entre a concentração determinada mediante aplicação do procedimento analítico 
e o nível de fortificação. Os valores de recuperação considerados adequados são estabelecidos em função da concentração do analito (20), conforme mostra a Tabela 1. Entretanto, de acordo com a área de pesquisa os valores aceitos podem variar. Essas diferenças podem ser observadas, até na mesma área, como no caso da análise de resíduos de pesticidas em que diferentes fontes estabelecem intervalo de aceitação de 70 a $120 \%$ (19), 80 a $110 \%$ (20) e 70 a $130 \%$ (21).

\section{TABELA1 - RECUPERAÇÃO DO ANALITO EM FUNÇÃO DA CONCENTRAÇÃO (35)}

\begin{tabular}{l|c}
\hline \multicolumn{1}{c|}{ Concentra a o do analito (\%) } & Intervalo de Recupera ${ }^{\circ}$ o Aceito (\%) \\
\hline$\geq 10$ & $98-102$ \\
$\geq 1$ & $97-103$ \\
$\geq 0,1$ & $95-105$ \\
$\geq 0,01$ & $90-107$ \\
$\geq 0,001-\geq 0,00001$ & $80-110$ \\
$\geq 0,000001$ & $60-115$ \\
$\geq 0,0000001$ & $40-120$ \\
\hline
\end{tabular}

A precisão costuma ser estimada em estudos intra-laboratoriais (repetitividade e precisão intermediária), usando-se os ensaios de recuperação, mediante cálculo dos coeficientes de variação (CV) (22). Em análise de resíduos de pesticidas considera-se que o método é preciso quando os coeficientes de variação apresentam-se menores que $20 \%$ (18). Isto deve-se, também, aos baixos níveis de concentração determinados.

O objetivo deste trabalho foi apresentar e discutir dois modos diferentes de avaliar os ensaios de recuperação em análise de resíduos de pesticidas, mediante estudo de casos. No primeiro, foram utilizados dados descritos na literatura para a análise de pesticidas organoclorados (OC) e organofosforados (OF) em amostras de água de coco por cromatografia a gás (CG) (23). No segundo caso foram estudados resultados de análise por cromatografia a líquido de alta eficiência (CLAE) do herbicida ácido 2,4-diclorofenoxiacético (2,4-D) e seu produto de degradação, o 2,4-diclorofenol (2,4-DCF), em amostras de solo (24). As estruturas químicas dos compostos estudados são apresentadas na Figura 1. 


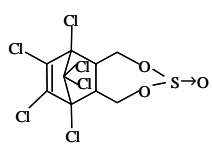

$\alpha$-Endosulfan<smiles>COP(=O)(OC)C(O)C(Cl)(Cl)Cl</smiles>

Triclorfon

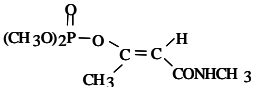

(E)

Monocrotof s<smiles>O=[SH]12CC3CC(O1)C(Cl)C(Cl)=C(Cl)C3(Cl)C2</smiles>

$\beta$-Endosulfan<smiles>CC1C2C=CC1C1C(=O)N(C)C(=O)C21</smiles>

Captan<smiles>CCOC(=O)CC(NP(C)(=O)OC)C(=O)OCC</smiles>

Malation

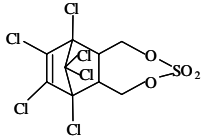

Sulfato de Endosulfan<smiles>Clc1ccc(Oc2cc(Cl)c(Cl)cc2Cl)cc1</smiles>

Tetradifon<smiles>CCOc1ccc([N+](=O)[O-])cc1</smiles>

Paration-metil

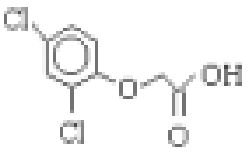

2,4-D

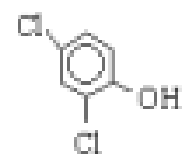

\section{ESTUDOS DE CASO: ENSAIO DE RECUPERAÇÃO DE PESTICIDAS}

O cálculo da recuperação para amostras fortificadas pode ser realizado computando-se as recuperações individuais para cada nível de fortificação do analito (i), avaliando-se a recuperação por meio de sua média (ii), ou analisando-se em 5 níveis a regressão linear da concentração obtida e a concentração de fortificação (iii) (22). Para ilustrar os itens i e iii foram usados dados da literatura para o cálculo das recuperações individuais de cada nível para organoclorados (OC) e organofosforados (OF), analisados por CG e de 2,4-D e 2,4-DCF analisados por CLAE.

\subsection{CASO 1: RESULTADOS OBTIDOS NA ANÁLISE DE OC E OF EM} ÁGUA DE COCO POR CG

Para avaliar a abrangência da exatidão foram estudados três níveis 
de fortificação e, para cada nível, efetuadas no mínimo cinco determinações (23). Os valores de recuperação obtidos para os pesticidas em estudo são apresentados na Tabela 2 e estão enquadrados nos limites (entre 70 e $120 \%$ ) descritos na literatura para resíduos de pesticidas.

\section{TABELA 2 - RECUPERAÇÕES PERCENTUAIS E COEFICIENTES DE VARIAÇÃO DOS COMPOSTOSORGANOCLORADOS (OC) E ORGANOFOSFORADOS (OF) ANALISADOS POR CG}

\begin{tabular}{|c|c|c|c|c|c|c|c|}
\hline Pesticidas & Classe & $\mathbf{n}$ & $\begin{array}{c}\mathrm{N} \text { vel } \\
\mathrm{mg} / \mathrm{Kg}\end{array}$ & $\begin{array}{c}\text { Recupera }{ }^{a} \text { o } \\
\text { M ødia (\%) }\end{array}$ & tcal & $\mathbf{S}$ & CV \% \\
\hline \multirow[t]{3}{*}{$\alpha$-Endosulfan } & $\mathrm{OC}$ & 6 & 0,01 & 95 & $-2,45$ & 4,6 & 4,8 \\
\hline & & 6 & 0,05 & 81 & $-3,58$ & 11,9 & 14,6 \\
\hline & & 8 & 0,1 & 91 & $-3,55$ & 6,7 & 7,4 \\
\hline \multirow[t]{3}{*}{$\beta$-Endosulfan } & $\mathrm{OC}$ & 6 & 0,01 & 99 & $-0,55$ & 4,0 & 4,1 \\
\hline & & 8 & 0,05 & 85 & $-3,58$ & 8,0 & 10,1 \\
\hline & & 8 & 0,1 & 94 & $-2,70$ & 5,9 & 6,2 \\
\hline \multirow[t]{3}{*}{ Captan } & $\mathrm{OC}$ & 6 & 0,1 & 91 & $-2,54$ & 7,9 & 8,7 \\
\hline & & 6 & 0,5 & 99 & $-0,50$ & 4,4 & 4,4 \\
\hline & & 6 & 1 & 104 & 3,05 & 2,9 & 2,8 \\
\hline \multirow[t]{3}{*}{ Malation } & OF & 5 & 0,5 & 93 & $-1,56$ & 8,9 & 9,6 \\
\hline & & 5 & 1,0 & 104 & 4,14 & 1,9 & 1,8 \\
\hline & & 6 & 2,0 & 99 & $-0,95$ & 2,4 & 2,4 \\
\hline \multirow[t]{3}{*}{ Monocrotof $\mathrm{s}$} & OF & 5 & 0,5 & 90 & $-2,46$ & 8,1 & 9,0 \\
\hline & & 5 & 1,0 & 95 & $-0,92$ & 10,9 & 11,5 \\
\hline & & 6 & 2,0 & 95 & $-1,87$ & 6,0 & 6,3 \\
\hline \multirow[t]{3}{*}{ Paration-metil } & OF & 5 & 0,5 & 97 & $-0,73$ & 8,2 & 8,5 \\
\hline & & 5 & 1,0 & 103 & 1,02 & 5,9 & 5,7 \\
\hline & & 6 & 2,0 & 99 & $-1,55$ & 1,4 & 1,4 \\
\hline Sulfato de & $O C$ & 6 & 0,01 & 95 & $-1,42$ & 7,9 & 8,3 \\
\hline \multirow[t]{2}{*}{ Endosulfan } & & 6 & 0,05 & 83 & $-4,50$ & 10,0 & 12,0 \\
\hline & & 8 & 0,1 & 94 & $-2,82$ & 5,6 & 6,0 \\
\hline \multirow{3}{*}{ Tetradifon } & $\mathrm{OC}$ & 5 & 0,5 & 80 & $-3,92$ & 10,19 & 12,74 \\
\hline & & 5 & 1,0 & 84 & $-3,81$ & 8,38 & 9,97 \\
\hline & & 6 & 2,0 & 97 & $-2,08$ & 3,22 & 3,32 \\
\hline \multirow[t]{3}{*}{ Triclorfon } & OF & 5 & 0,5 & 80 & $-4,57$ & 8,75 & 10,63 \\
\hline & & 5 & 1,0 & 85 & $-3,46$ & 8,59 & 10,10 \\
\hline & & 6 & 2,0 & 98 & $-1,59$ & 2,81 & 2,87 \\
\hline
\end{tabular}

$\mathrm{n}$ = número de análises; $\mathrm{S}=$ Variância; CV = Coeficiente de Variação; $t 4,99 \%=4,6041$, $\mathrm{t} 5,99 \%=4,0321, \mathrm{t} 7,99 \%=3,4995$.

Fonte: BRITO et al., 2002 (23).

Para confirmar a exatidão do método, os resultados foram submetidos ao teste de hipótese, sendo estabelecida como hipótese nula $\left(\mathrm{H}_{0}\right)$ : Rec. $=100 \%$ e como hipótese alternativa $\left(\mathrm{H}_{1}\right)$ : Rec. ${ }^{1} 100 \%$. O teste $\mathrm{t}$ aplicado, com $99 \%$ de confiança e n-1 graus de liberdade, apresentou valores calculados entre os intervalos estabelecidos pelo valor tabelado. 
Deste modo, não se poderia afirmar a existência de diferenças significativas entre as recuperações obtidas e o valor esperado (100\%), considerando -se que o método proposto fosse suficientemente exato. Utilizou-se, assim, a equação:

$$
t_{\text {calculado }}=\frac{(\overline{\operatorname{Rec}}-100)}{\frac{s}{\sqrt{n}}} \quad \text { Eq. } 1
$$

na qual:

$\overline{\operatorname{Re}}$ c é a recuperação média; 100 é a recuperação desejada; sé o desvio-padrão das recuperações; $n$ é o número de replicatas (grau de liberdade igual a $n$ para este estudo).

Aceita-se a hipótese nula quando o valor absoluto de tcalculado for menor que o valor tabelado de $t$ (18). A utilização de intervalo de confiança amplo (99\% de confiança) mostrou-se indicada, uma vez que o estudo foi realizado em níveis de concentração na ordem de $\mu \mathrm{g} / \mathrm{L}$. O uso de intervalo mais restrito (90 ou 95\%) aumentaria a probabilidade de ocorrer erro tipo "falso negativo", isto é, poder-se-ia rejeitar resultado que na realidade é verdadeiro (18).

Para o estudo de precisão, os coeficientes de variação (obtidos a partir dos valores referentes ao ensaio de recuperação) são considerados adequados quando iguais ou inferiores a $20 \%$, conforme recomendado por THIER e ZEUMER (19). Como todos os valores de CV mostraram-se inferiores ao estabelecido, o método proposto foi considerado preciso.

\subsection{CASO 2: RESULTADOS OBTIDOS NA ANÁLISE DE 2,4-D E 2,4- DCF EM SOLOS POR CLAE}

Os resultados de recuperação e os respectivos CV obtidos na análise de 2,4-D e 2,4-DCF em solos são apresentados na Tabela 3. Observou-se que os valores obtidos para todos os níveis de fortificação enquadraram-se na faixa estabelecida para a análise de resíduos de pesticidas (18). Adicionalmente, aplicou-se o teste de hipóteses com a finalidade de demonstrar a inexistência de diferença significativa entre os valores obtidos e o esperado (100\%). Para este estudo foram estabelecidas as hipóteses nula e alternativa como no caso anterior. 
Com $99 \%$ de confiança e 4 graus de liberdade a hipótese nula é aceita e não se pode afirmar se há diferença significativa entre os valores obtidos e o esperado. Utilizando intervalo de probabilidade mais restrito (95\% de confiança) quatro dos níveis para 2,4-D e dois para 2,4-DCF apresentaram valores absolutos de $\mathrm{t}$ superiores ao valor tabelado, rejeitando-se nesse nível de confiança a hipótese nula. Esses resultados confirmam o que foi citado anteriormente com relação ao intervalo de confiança em função do nível de concentração. Assim, os valores encontrados não podem ser considerados significativamente diferentes do valor desejado.

\section{TABELA 3 - RECUPERAÇÕES PERCENTUAIS E COEFICIENTES DE VARIAÇÃO OBTIDOS NA ANÁLISE DE 2,4-D E 2,4-DCF POR CLAE}

\begin{tabular}{|c|c|c|c|c|c|c|}
\hline Composto & $\begin{array}{c}\mathrm{N} \text { vel de } \\
\text { fortifica a o } \\
(\mathrm{mg} / \mathrm{Kg})\end{array}$ & $\begin{array}{c}\text { Recupera }{ }^{\mathrm{a}} \mathrm{o} \\
(\%)\end{array}$ & Desvio-padr ${ }^{\mathrm{a}} 0$ & $n$ & $\begin{array}{l}\text { CV } \\
\text { (\%) }\end{array}$ & $t_{\text {calculado }}{ }^{*}$ \\
\hline 2,4-D & $\begin{array}{l}4,0 \\
3,0 \\
2,0 \\
1,0 \\
0,1\end{array}$ & $\begin{array}{c}89 \\
95 \\
98 \\
104 \\
111\end{array}$ & $\begin{array}{l}6,2 \\
4,3 \\
2,6 \\
3,1 \\
5,6\end{array}$ & $\begin{array}{l}5 \\
5 \\
5 \\
5 \\
5\end{array}$ & $\begin{array}{l}7,0 \\
4,5 \\
2,6 \\
3,0 \\
5,0\end{array}$ & $\begin{array}{c}-3,97 \\
-2,60 \\
-1,72 \\
2,88 \\
4,39\end{array}$ \\
\hline 2,4-DCF & $\begin{array}{l}4,0 \\
3,0 \\
2,0 \\
1,0 \\
0,1\end{array}$ & $\begin{array}{c}101 \\
99 \\
98 \\
96 \\
95\end{array}$ & $\begin{array}{l}3,5 \\
4,1 \\
2,9 \\
2,9 \\
3,8\end{array}$ & $\begin{array}{l}5 \\
5 \\
5 \\
5 \\
5\end{array}$ & $\begin{array}{l}3,4 \\
4,1 \\
3,0 \\
3,0 \\
4,0\end{array}$ & $\begin{array}{c}0,64 \\
-0,54 \\
-1,54 \\
-3,08 \\
-2,94\end{array}$ \\
\hline
\end{tabular}

* Para $n=5$, t95\% = 2,78 e t99\% = 4,60 (11); CV = Coeficiente de Variação.

Fonte: AMARANTE Jr. et al., 2002 (24).

Os desvios-padrão relacionados a cada nível de fortificação são apresentados na Figura 2. Observou-se para o 2,4-D tendência do desviopadrão em diminuir no centro do intervalo de trabalho, sugerindo que as concentrações extremas aproximaram-se do limite de linearidade do método. Para o 2,4-DCF os desvios-padrão de todos os níveis apresentaram-se similares, com exceção do nível de fortificação de $3,0 \mathrm{mg} / \mathrm{kg}$. Tal efeito pode sugerir erros analíticos. 


\section{FIGURA 2 - RESULTADOS DE RECUPERAÇÃO EM FUNÇÃO DO NÍVEL DE FORTIFICAÇÃO E SEUS RESPECTIVOS DESVIOS- PADRÃO}
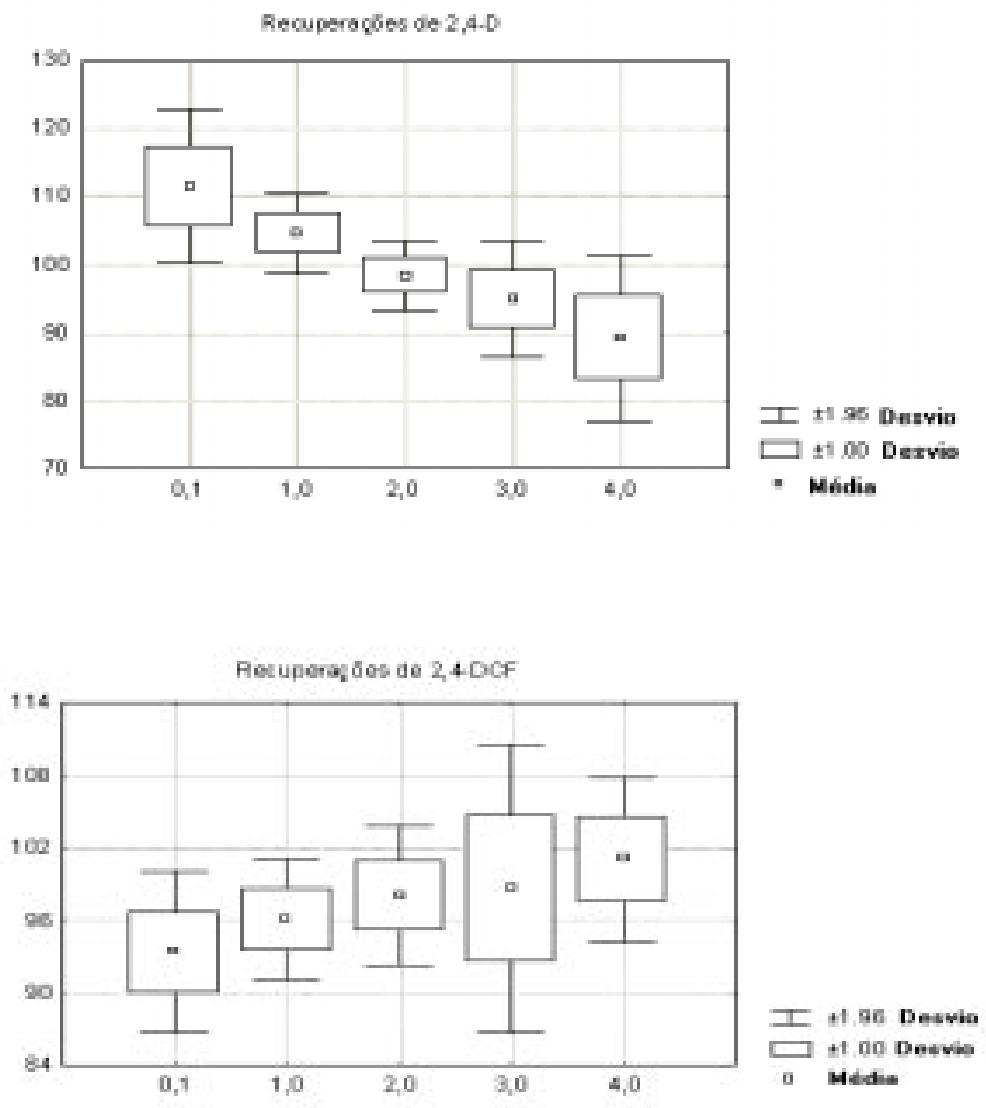

Outro parâmetro de validação pode ser analisado pelos dados de recuperação, ou seja, a seletividade do método. Nesse caso, avalia-se a correlação entre a concentração obtida e a concentração de fortificação (12). Pode-se realizar teste de hipótese, comparando os valores do coeficiente linear ( $\mathrm{c} 0$ ) e coeficiente angular ( $\mathrm{c} 1$ ) com os valores esperados (zero e um, respectivamente). Neste estudo, considerou-se " $n-2$ " graus de liberdade, usando-se as equações: 
Eq. $2 \quad t=\frac{\left|c_{0}-0\right|}{\frac{s_{c_{0}}}{\sqrt{n-2}}}$

Eq. $3 \quad t=\frac{\left|c_{1}-1\right|}{\frac{s_{c_{1}}}{\sqrt{n-2}}}$

Nas quais:

t é o t calculado;

$\mathrm{C}_{0}$ é o coeficiente linear;

$\mathrm{C}_{1}$ é o coeficiente angular;

$\mathrm{S}_{\mathrm{c} 1}$ é o desvio-padrão do coeficiente angular;

$\mathrm{S}_{\mathrm{co}}$ é o desvio-padrão do coeficiente linear (18).

3 e Tabela 4.

Os resultados referentes a este estudo são apresentados na Figura

FIGURA 3 - LINEARIDADE DA RELAÇÃO ENTRE A CONCENTRAÇÃO RECUPERADA E A CONCENTRAÇÃO DE FORTIFICAÇÃO

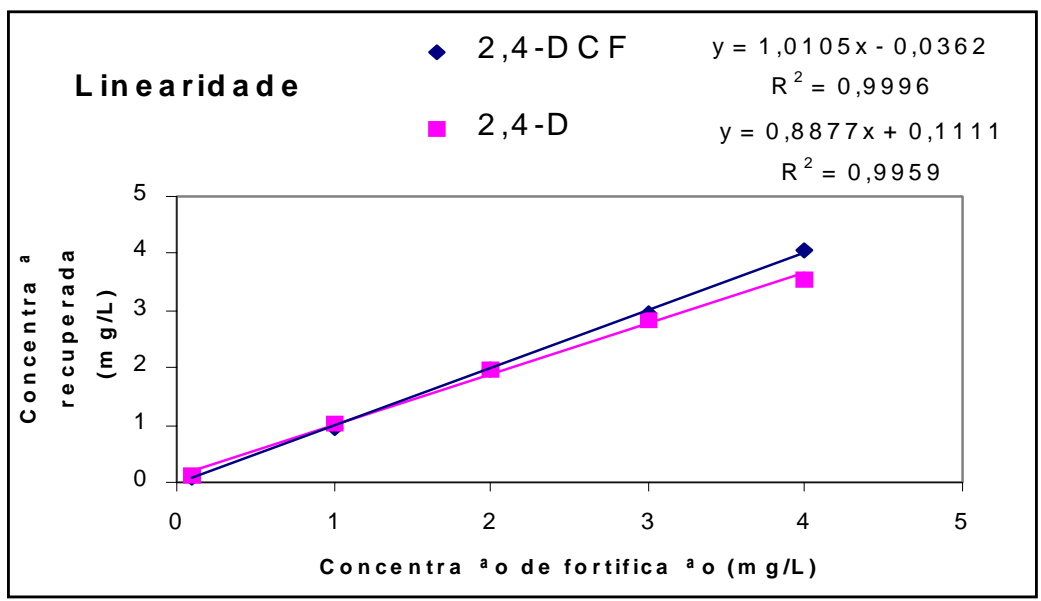

Observou-se, pelos coeficientes de correlação, que a relação entre as concentrações de fortificação e os valores recuperados é 
suficientemente linear, para assegurar o intervalo de trabalho escolhido. Entretanto, para averiguar o efeito dos compostos presentes na matriz é necessário observar os valores dos coeficientes linear e angular. A hipótese nula foi aceita (Tabela 4) para ambos os coeficientes, com 99\% de confiança e 3 graus de liberdade (tanto para 2,4-D e 2,4-DCF). Desta forma, não se pode dizer que existam diferenças significativas entre os coeficientes e os valores esperados, indicando que os componentes da amostra não interferiram na determinação dos compostos de interesse.

\section{TABELA 4- RESULTADOS REFERENTES AO ESTUDO DA LINEARIDADE ENTRE A CONCENTRAÇÃO RECUPERADA E A CONCENTRAÇÃO DE FORTIFICAÇÃO}

\begin{tabular}{|c|c|c|c|}
\hline & $\mathbf{C}_{0}$ & $C_{1}$ & $\mathbf{t}_{\text {calculado }}$ \\
\hline \multirow{5}{*}{$2,4-D$} & 0,0094 & 0,9735 & \multirow[t]{2}{*}{$\mathrm{C}_{0}: 3,05$} \\
\hline & 0,1311 & 0,8629 & \\
\hline & 0,1194 & 0,8663 & \multirow[t]{3}{*}{$c_{1}:-3,71$} \\
\hline & 0,1202 & 0,8814 & \\
\hline & 0,1871 & 0,8257 & \\
\hline Mødia & 0,11344 & 0,88196 & \\
\hline Desvio & 0,064504 & 0,055122 & \\
\hline \multirow{5}{*}{$2,4-\mathrm{DCF}$} & $-0,0237$ & 1,0136 & $c_{0}:-2,07$ \\
\hline & $-0,0701$ & 1,0496 & \multirow{4}{*}{$c_{1}: 0,56$} \\
\hline & 0,0085 & 0,9655 & \\
\hline & $-0,0406$ & 1,0088 & \\
\hline & $-0,0563$ & 1,0112 & \\
\hline Mødia & $-0,03644$ & 1,00974 & \\
\hline Desvio & 0,030523 & 0,029849 & \\
\hline
\end{tabular}

$(99 \%)=5,8409 ; \mathrm{t}(95 \%)=3,1825$.

\section{CONCLUSÃO}

A validação de métodos analíticos é necessária para o reconhecimento das limitações e confiabilidade dos resultados obtidos. 
O uso de métodos validados é essencial para obtenção de resultados confiáveis que possam ser satisfatoriamente interpretados. A exatidão e a precisão são as chaves do processo de validação de métodos analíticos. Diferentes procedimentos podem ser adotados para a avaliação da exatidão, sendo os ensaios de recuperação os mais empregados por não dependerem de fatores externos para serem realizados.

Nos casos relatados foram obtidas recuperação entre 80 e 104\% e Coeficientes de Variação entre 2,81 e 12,74\%, ambos enquadrados na faixa recomendada pela Association of Official Analytical Chemists (AOAC) para os níveis de concentração investigados. Os estudos evidenciaram que os ensaios de recuperação são adequados para a avaliação dos critérios de exatidão e precisão, para as mesmas matrizes.

\begin{abstract}
EVALUATION OF METHODS ACCURACY AND PRECISION FOR ANALYSIS OF PESTICIDES RESIDUES THROUGH RECUPERATION ASSAYS

The importance of accuracy and precision in the methods validation of pesticides residues analysis is discussed. The main processes for accuracy evaluation are presented through two case studies. In the cited cases, recoveries were obtained between 80 and $104 \%$ and variation coefficient between 2,81 and $12,74 \%$ both in the range recommended by AOAC for the concentration levels investigated. The studies evidenced that the recovery assays were adequate for the evaluation of the accuracy and precision criteria for the same matrix.

KEY-WORDS:METHODS OF ANALYSIS-VALIDATION, ANALYSIS, PESTICIDES.
\end{abstract}

\title{
REFERÊNCIAS
}

1 POLESELLO, S. How to present an analytical method. Food Chem., v. 58, n. 1/2, p. 145-147, 1997.

2 HUBERT, P.H.; CHIAP, P.; CROMMEN, J. et al. The SFSTP guide on the validation of chromatographic methods for drug bioanalysis: from the Washington Conference to the Laboratory. Anal. Chim. Acta, Amsterdã, v. 391, p. 135-148. 1999.

3 CAUSON, R. Validation of chromatographic methods in biomedical analysis: viewpoint and discussion. J. Chromatogr. B., v. 689, p. 175, 1997.

4 WIELING, J. et al. Rational experimental design for bioanalytical methods validation: illustration using an assay method for total 
captopril in plasma. J. Chromatogr. A., v. 730, p. 381. 1996.

5 BRESSOLLE, F.; BROMET-PETIT, M.; AUDRAN, M. Validation of liquid chromatographic and gas chromatographic methods: applications to pharmacokinetics. J. Chromatogr. B., v. 686, p. 3, 1996.

6 CHASIN, A. M.; CHASIN, M.; SALVADORI, M. C. Validação de métodos cromatográficos em análises toxicológicas. R. Farm. Bioquim., v. 30, n. 2, p. 49-53, 1994.

7 FRANCOTTE, E.; DAVATZ, A.; RICHERT, P. Development and validation of chiral high-performance liquid chromatographic methods for the quantification of valsartan and to the tosylate of valinebenzyl ester. J. Chromatogr. B., v. 686, p. 77, 1996.

8 McDONALD, R. D. The role of laboratory information management system (LIMS) in analytical method validation. Anal. Chim. Acta., v. 391, p. 149, 1999.

9 CURRIE, L. A.; SVEHLA, G. Nomenclature for the presentation of results of chemical analysis. Pure \& Appl. Chem., v. 66, p. 595, 1994.

10 PEREIRA, D. M. C.; ARCOS, M. A. S. V.; AMARANTE Jr., O. P. de; CALDAS, E. P. A. Comparação de métodos dicromatométricos para a determinação de ferro total em minérios de ferro. Anais Assoc. Bras. Quím., São Paulo, v. 49, p. 198-203, 2000.

11 FEINBERG, M.; RAGUÈNÈS, N. Development and application of standardized validation procedure for food chemistry laboratories. Anal. Chim. Acta., Amsterdã, v. 391, p. 239-252, 1999.

12 VALIDATION of analytical methods: definitions and terminology. CPMP/ICH/381/95. London: $\mathrm{ICH}, 1995.5$ p. (ICH Harmonised Tripartite Guideline).

13 VALIDATION of chromatography methods: review guidance. London: Center for drug evaluation and research (CDER), 1994. $30 \mathrm{p}$. 
14 ANALYTICAL procedure and methods validation: chemistry, manufaturing, and controls documentation. London: Guidance for Industry, 2000.

15 INTERNATIONAL CONFERENCE ON HARMONISATION OF TECHINICAL REQUERIMENTS FOR REGISTRATION OF FHARMACEUTICALS FOR HUMAN USE. Text on validation of analytical procedures. [London], 1994. 5 p. (ICH harmonised tipartite guideline). Disponível em: <http:// www.ich.org/ich1.html>.

16 INTERNATIONAL CONFERENCE ON HARMONISATION OF TECHINICAL REQUERIMENTS FOR REGISTRATION OF FHARMACEUTICALS FOR HUMAN USE. Validation of analytical procedures: metodology. [London], 1996. 8 p. (ICH Harmonised Tipartite Guideline). Disponível em: <http:// www.ich.org/ich1.html>.

17 INTERNATIONAL CONFERENCE ON HARMONISATION. Validation of analytical procedures: metodology. London, 1996. 9 p. (ICH Harmonised Tripartite Guideline). CPMP/ICH/ $281 / 95$.

18 AMARANTE Jr., O. P. de; CALDAS, E. P. A.; BRITO, N. M.; SANTOS, T. C. R. dos; VALE, M. L. B. F. Validação de métodos analíticos: uma breve revisão. Cad. Pesq., São Luís, v. 12, n.1/ 2, p. 116-131, 2001.

19 THIER, H. P.; ZEUMER, H. Manual of pesticide analysis. New York: Verlag Chemie, 1987. p. 37-41.

20 AOAC. Association of Official Analytical Chemists. Peer verified method program: manual on polices and procedures. Arlington, VA, 1993.

21 AMARANTE Jr., O. P. de. Avaliação do potencial de contaminação por herbicidas: determinação do 2,4-D e do seu principal produto de degradação em solos de campos de cultivo de eucaliptos. São Luís, 2002. 125 p. Dissertação (Mestrado em Química Analítica), Departamento de Tecnologia Química, Universidade Federal do Maranhão. 
22 INTRA-LABORATORY testing of method accuracy from recovery assays. Talanta, v. 48, p. 729, 1999.

23 BRITO, N. M.; NAVICKIENE, S.; POLESE, L.; JARDIM, E.; ABAKERLI, R.; RIBEIRO, M. L. Determination of pesticide residues in coconut water by liquid-liquid extraction and gas chromatography with electron-capture plus termionic specific detection and solid-phase extraction and high-performance liquid chromatography with ultraviolet detection. J. Chromatogr. A., v. 957, p. 201, 2002.

24 AMARANTE Jr., O. P. de; BRITO, N. M.; SANTOS, T. C. R. dos; RIBEIRO, M. L. Determination of 2,4-dichlorophenoxyacetic acid $(2,4-D)$ and its major transformation product in soil samples by liquid chromatographic analysis. Talanta, 2003. [no prelo]. 